\title{
Learning and Memory Is Reflected in the Responses of Reinforcement-Related Neurons in the Primate Basal Forebrain
}

\author{
F. A. W. Wilson and E. T. Rolls \\ Department of Experimental Psychology, University of Oxford, Oxford OX1 3UD, United Kingdom
}

\begin{abstract}
Certain basal forebrain neurons encode the learned reinforcement value of objects: they respond differentially to visual stimuli that signal availability of fruit juice (positively reinforcing) or saline (negatively reinforcing) obtained by lick responses in visual discrimination tasks. In this report we describe the rapid, learning-related changes in the responses of these neurons during the acquisition and reversal of the reinforcement contingency of a visual discrimination reversal task. The same neurons also responded differentially to novel and familiar stimuli in 2 recognition memory tasks, in which monkeys applied the learned rule that lick responses to novel stimuli elicited saline and responses to familiar stimuli elicited juice. These differential responses to novel and familiar stimuli thus reflected the reinforcement value of the stimuli. A single presentation of a novel or a familiar stimulus was sufficient to elicit a differential response which was maintained even when the stimulus had not been seen recently. The maintenance of the differential response indicates that these neurons are influenced by a durable memory for the stimuli, estimated to be 30 trials on average. These differential neurons were recorded in the substantia innominata, the diagonal band of Broca, and a periventricular region of the basal forebrain. The responses of the reinforcement-related neurons in these 3 regions were similar in most respects. These results support the conclusion that basal forebrain neurons respond to sensory stimuli that, through learning of different contingencies, signal the availability of reinforcement. We suggest that the properties of learning and memory reflected in these neuronal responses are due to afferent pathways from ventromedial regions of the prefrontal and temporal cortices and the amygdala, and that the responses of these neurons provide an enabling mechanism that facilitates the operation of diverse cortical regions in which specific sensory, motor, or mnemonic functions take place.
\end{abstract}

There is a wealth of evidence that the basal forebrain is involved in the control of motivated behaviors such as feeding and drinking, and this evidence strongly suggests that neurons in the basal forebrain make a significant contribution to the substrate of reinforcement (Wilson and Rolls, 1989a). Other forms of evi-

Received May 22, 1989; revised Sept. 25, 1989; accepted Oct. 12, 1989.

This work was supported by the Medical Research Council of Great Britain. F.A.W.W. gratefully acknowledges the award of an M.R.C. studentship during this research. We would like to thank Dr. Colleen Phillips for her participation in some of the experiments.

Correspondence should be addressed to Fraser Wilson, Section of Neuroanatomy, Yale University School of Medicine, 333 Cedar Street, New Haven, CT 06510.

Copyright (C) 1990 Society for Neuroscience $0270-6474 / 90 / 041254-14 \$ 02.00 / 0$ dence indicate that the basal forebrain plays a role in cognitive processes, for dementia and impairments of memory and attention have been reported to follow damage involving the basal forebrain and adjacent cortical regions in man (Friedman and Allen, 1969; Gascon and Gilles, 1973; Damasio et al., 1985a, b). It is known that lesions of the anterior third ventricle that produce memory deficits also produce changes in drinking behavior (Nichelli et al., 1982), suggesting that the basal forebrain contributes both to the generation of motivated behavior and memory function. The involvement of the cholinergic basal forebrain in aspects of cognitive function is also indicated by 2 observations on the brains of patients with Alzheimer's disease: a decrease in the cholinergic marker enzyme choline acetyltransferase in the cerebral cortex (Bowen et al., 1976; Davies and Maloney, 1976; Perry et al., 1977); and a loss of cells in the basal nucleus of Meynert within the basal forebrain (Whitehouse et al., 1982).

However, there are difficulties in attributing the cognitive deficits of Alzheimer's disease solely to the degeneration in the basal forebrain. First, pathological changes in the brains of patients with Alzheimer's disease are known to occur in cortical and subcortical structures as well as the basal forebrain (Bondareff et al., 1982; Brun and Englund, 1981), and thus damage to these other structures may be responsible for the deficits in Alzheimer's disease. Second, a role for the basal forebrain in processes such as the initiation and termination of feeding and drinking does not appear to be reflected in the deficits of Alzheimer's disease, which are cognitive rather than regulatory or vegetative in nature.

In order to understand more clearly the relationship between the motivational and cognitive processes occurring in the basal forebrain, and thus how damage to this subcortical region might influence cognitive behavior, the present experiments examined the responses of basal forebrain neurons in monkeys performing memory tasks. Previous work in the lateral hypothalamus and substantia innominata has shown that neurons in these structures respond differently to visual stimuli that monkeys have learned are used to deliver fruit juice or saline (Mora et al., 1976; Rolls et al., 1979). We have established that the properties of visual discriminanda such as their shape, size, and color are not the basis of these differential neuronal responses by recording from basal forebrain neurons in monkeys performing 2 different visual discrimination tasks (Wilson and Rolls, 1989a). In the present experiments, we examined the rcsponses of these neurons during the acquisition and reversal of a third discrimination task when the monkeys had to learn about changes in reinforcement contingencies. The monkeys also performed recognition memory tasks and had to remember the novelty and familiarity of visual stimuli in order to determine their rein- 
forcement value. These tasks require judgments of stimulus familiarity, similar to tasks in which patients with Alzheimer's disease are impaired (Albert and Moss, 1984). Specifically, we studied the way in which learning and memory are reflected in the activity of neurons that responded to reinforcing stimuli such as foods and liquids that monkeys will work to obtain. We found that irrespective of the appearance of the stimuli, and even when a rule based on whether a stimulus is novel or familiar must be used to determine its reinforcement value, basal forebrain neurons respond to stimuli that, through learning, signal the availability of reinforcement and reflect access to a durable memory for the stimuli. Some of the data presented here have been published in abstract form (Wilson et al., 1984).

\section{Materials and Methods}

Subjects, stimulus presentation, behavioral responses, and reinforcement. Two $M$. mulatta and $2 M$. fascicularis monkeys were trained to perform visual discrimination tasks; the 2 rhesus monkeys also performed recognition memory tasks. When sitting in the primate chair, the monkeys' view of the laboratory was limited to a circular aperture in an enclosure that surrounded the chair. Head fixation and the enclosure ensured that the field of view was restricted to visual stimuli presented in the aperture. The aperture allowed different types of visual stimuli to be presented: (1) 3-dimensional objects were presented using a $6.4 \mathrm{~cm}$ aperture electromagnetic shutter mounted on the enclosure 8-12 inches from the monkey; (2) video images were presented on a monitor screen and viewed through the aperture, and (3) objects and foods were presented and delivered to the monkey through the aperture. A tube mounted in front of the mouth delivered saline or juice reinforcement, dependent upon the behavioral responses. During the performance of the tasks, a tone cue of $500 \mathrm{msec}$ duration preceded the visual stimuli, facilitating fixation of the stimuli. Visual stimuli were presented for $1.5 \mathrm{sec}$ and the intertrial interval was generally $6 \mathrm{sec}$, or $8 \mathrm{sec}$ for selected experiments. Lick responses in the intertrial interval resulted in the delivery of saline.

The monkeys were fed fruit and nuts throughout the experiment, and drank juice obtained through task performance. Laboratory chow and ad libitum water was available after their return to their home cage. The monkeys gained weight steadily during the course of the experiments.

The visual discrimination and visual discrimination reversal tasks. Two visual discrimination tasks were performed by the monkeys. In the visual discrimination shutter-based task (VDS), an electromagnetic shutter was used to present 2 highly familiar syringes mounted in square plaques of different colors, one per trial. Lick responses at the presentation of a black syringe (the $S-$ ) resulted in the delivery of aversive hypertonic saline, while responses to the white syringe $(\mathrm{S}+$ ) resulted in the delivery of rewarding fruit juice. In the visual discrimination computer-based task (VDC), 2 visual images equated for size, color and brightness, but differing in shape were displayed on a video monitor. Lick responses to the yellow circle $(\mathbf{S}+)$ produced fruit juice, while responses to the yellow square produced saline. The monkeys learned to respond differentially to the $S+$ and $S-$ stimuli in the 2 tasks.

In the visual discrimination reversal task, the stimuli were 2 plaques, varying in color and shape. Inserted in the plaques were syringes which were used to deliver fruit juice or saline during training. The stimuli were hand-held, but only the plaques were visible when presented with the electromagnetic shutter against a white background. The reversal task was generally used only once per day (i.e., to test a differential neuron), and so during this test the stimulus had not been seen for 18 hr. During this type of experiment, a lick response to the first of the 2 stimuli in the set to be presented was always reinforced with juice. By convention, lick responses to the other subsequently presented stimulus resulted in saline. After a minimum of 6 pseudorandomly interdigitated presentations of each stimulus in which correct responses were made, the reinforcement value of these stimuli was changed, so that the positive stimulus became negative and the negative became positive. The cue that the reinforcement contingency had changed was simply that the response to the formerly positive stimulus resulted in the delivery of saline. Daily practice with this task resulted in reversals of the behavioral responses within 3 presentations of a stimulus after the change in the reinforcement contingency.
The recognition memory tasks. In the serial visual recognition memory task, lick responses during the presentation of novel stimuli elicited aversive saline, while lick responses to familiar stimuli resulted in the delivery of rewarding fruit juice. Thus, the monkeys had to determine the novelty or familiarity of the stimuli in order to obtain juice. The first (novel) presentation of a stimulus was followed by a second (familiar) presentation of the stimulus after $0-16$ other trials, selected in pseudorandom order. A typical stimulus sequence was as follows: $\mathrm{N} 1 \rightarrow \mathrm{N} 2 \rightarrow \mathrm{F} 2 \rightarrow \mathrm{N} 3 \rightarrow \mathrm{N} 4 \rightarrow \mathrm{F} 1 \rightarrow \mathrm{N} 5 \rightarrow \mathrm{F} 4$. The novel stimulus (N1) shown on trial 1 was shown again after 4 intervening trials as familiar (F1) on trial 6 , while the novel stimulus (N2) shown on trial 2 was repeated with no intervening trials on trial 3.

Two versions of the recognition memory tasks were learned. In the recognition memory shutter-based task (RMS), 3-dimensional objects were presented using the electromagnetic shutter. Stimuli were handheld, but only the objects were visible against a white background. A given object, drawn from a population of approximately 2000 objects, was presented twice daily, once as novel and once as familiar. The entire stimulus set was presented once every $2-4$ weeks. Thus, a novel stimulus is operationally defined as a stimulus not seen for 14 or more days. The monkeys responded to stimuli that had not been seen for 2 weeks as if they were novel. Their performance on the recognition task was on average better than $90 \%$ correct.

In the recognition memory computer-based task (RMC), 2-dimensional colored visual images were presented on a monitor screen (the RMC task) viewed through the aperture in the primate chair. The video images ( $n=607$ ) were digitized, stored on computer disk, and displayed using a Matrox QRGB framestore. The images were seen twice monthly before replacement.

Recording techniques. Neuronal activity was recorded using glassinsulated tungsten microelectrodes, which were advanced with a hydraulic microdrive mounted on an implanted stainless steel chamber. The signal was fed to an FET buffer amplifier, filtered, amplified, and displayed on an oscilloscope. Neuronal activity was discriminated with the trigger circuit of an oscilloscope and converted to digital pulses. A PDP-11 computer sampled and displayed neuronal activity on successive trials in the form of a dot display, relative to the onset of the task stimuli. EOG data were usually sampled at $100 \mathrm{~Hz}$, digitized, and stored with neuronal activity on computer disk and magnetic tape. Chronically implanted electrodes were used to deliver single-pulse electrical stimulation through constant-current stimulus isolation devices to determine possible connections between the basal forebrain, cortex, and thalamus.

General experimental procedure and localization of the recording sites. During the recordings the monkeys continuously performed the different tasks. The presentation of novel and familiar objects, and stimuli in a visual discrimination task (the $S_{+}$and the $S_{-}^{-}$) were interdigitated in pseudorandom order. Each neuron was tested for responsiveness to novel and familiar stimuli, and to the $S+$ and $S-$. If the neuron responded to the presentation of any of these stimuli, extensive testing for periods of up to $4 \mathrm{hr}$ continued in order to identify the properties of the stimuli that elicited the responses. A record of the depth of each neuron was made, as well as its response properties or lack of responsiveness. Every neuron logged during these experiments forms part of the data base of this study. After recording from a basal forebrain neuron, the electrode was advanced by a minimum of $100 \mu \mathrm{m}$ before sampling other neurons. Sampling of neuronal activity in the cortex, basal ganglia, and amygdala provided control data with which neurons in the basal forebrain could be compared.

Recordings of single neuron activity began when the electrode penetrated the cortex; collection of data began at a depth of $15 \mathrm{~mm}$ from the cortical surface. This sampling resulted in a profile of the brain structures through which the electrode passed, which aided localization of the basal forebrain as traversal of the internal capsule and anterior commissure resultcd in cessation of ncuronal activity and provided a guide to the proximity of the basal forebrain. Short-latency eye-movement-related activity from the optic chiasm and tract indicated that the electrode had left the substantia innominata. As the recording electrode passed from the diagonal band of Broca and approached the skull, an increase in the background noise of the recording system was observed, serving as a further aid to localization.

At the completion of each experiment, X-radiographs in the coronal and parasaggital planes were taken of the skull and the microelectrode in situ. Small lesions were made at the site of selected neurons by passing DC current (80-100 $\mu \mathrm{A}$ for $80 \mathrm{sec})$ through the recording electrode. These lesions were made in a 3 week period prior to perfusion, were 
often made at the sites of responsive neurons, and were targeted to bracket the brain regions in which the recordings took place. The lesions were also used to determine brain shrinkage due to perfusion by making 2 lesions per track at a known distance apart. After sacrifice the skull was mounted in a stereotaxic frame, and a sharpened hollow tube was passed through the brain parallel to the plane formed by the inferior orbital ridge and external auditory meatus, and an X-ray was taken, providing a further reference; the brain was blocked in the stereotaxic vertical plane. Frozen sections in the coronal plane were cut and collected every $50 \mu \mathrm{m}$ and stained with cresyl violet. The sections were examined to determine the location of electrode tracks, microlesions, and the implanted stimulation electrodes. A linear regression was performed in each of the 3 dimensions between measurements in the brain and measurements of the radiographs of the positions of the lesions and electrode tracks. The accuracy of reconstruction according to this method was better than $0.5 \mathrm{~mm}$, as shown by the standard deviation of the intercepts, and the correspondence between the locations in which neurons were not recorded, and the reconstructed positions of the internal capsule, anterior commissure, optic tract, and ventricles.

Large-scale $(\times 10)$ drawings were made of the brain sections. Plots $(\times 10)$ were made of the positions of the electrode tracks every $0.5 \mathrm{~mm}$ from the X-radiograph measurements, corrected for the expansion in the $X$-ray negative. By superimposing the 2 sets of drawings, the location of each neuron could be reconstructed, relative to implanted stimulation electrodes and microlesions.

Data analysis. For each trial of the memory tasks, the computer counted the number of spikes emitted over a $500 \mathrm{msec}$ period, starting $100 \mathrm{msec}$ after the stimulus onset. Data for the different trials (novel, familiar, $\mathrm{S}+, \mathrm{S}-$ ) were compared using 1 -way analysis of variance and subsequent Tukey tests (Bruning and Kintz, 1977). The comparisons between novel and familiar stimuli were based on data collected from between 6 and 164 novel stimuli for each neuron. All the differential responses cited are significant $(p<0.05)$, the majority being significant at $p<0.01$ )

Scatter plots are used to represent the responses of differential neurons. This technique is used in order to show how each neuron responded in the various conditions. The data points were calculated by determining the spontaneous firing rate of a neuron, and then subtracting this value from the responses elicited by the stimuli. Thus, the data points represent increases or decreases in firing rate from the baseline activity, represented by the axes.

The latencies at which neurons responded differentially to novel and familiar stimuli were determined with the use of cumulative sum techniques (Woodward and Goldsmith, 1964) implemented on a computer. Peristimulus time histograms were computed for each type of trial (e.g., to novel and familiar stimuli), and subtracted from each other; the cumulative sum of this difference array was then calculated to allow estimation of the differential response latency.

\section{Results}

The present experiments were carried out on 120 reinforcementrelated neurons recorded in (1) the substantia innominata (73/ $1058)$, a region ventral to the globus pallidus and putamen, and lateral to the base of the internal capsule; (2) the diagonal band of Broca (24/489), a region abutting the base of the brain anterior to the optic chiasm and within $4 \mathrm{~mm}$ of the midline at its basal portion; and (3) the periventricular region (23/572), which incorporates part of the anterior hypothalamus and adjacent structures between the wall of the third ventricle and the internal capsule, and between the anterior thalamus and anterior commissure. These neurons responded on the basis of the reinforcement value of a range of visual and auditory stimuli (Wilson and Rolls, 1989a). For example, they responded differentially to the $\mathrm{S}+$ and $\mathrm{S}-$ stimuli in 2 visual discrimination tasks. Two types of reinforcement-related neurons were found: $S+$ neurons responded to rewarding stimuli, while $S-$ neurons responded to aversive stimuli. This terminology refers to the stimulus in the discrimination task to which the differential neuron was most responsive.

\section{The visual discrimination reversal task}

The differential responses observed in the 2 visual discrimination (VDS and VDC) tasks showed that the responses of these basal forebrain neurons reflected the learned reinforcing properties of 2 different sets of stimuli, which prior to training in the tasks had no relationship to reinforcement.

In order to examine neuronal activity during learning more directly, reinforcement-related neurons in the substantia innominata (SI) and diagonal band of Broca (DBB) were tested during the performance of a third visual discrimination task and its reversal, in which the monkey had to learn the current reinforcement value of the stimuli, and the change in reinforcement value after reversal (see Materials and Methods). The reversal task was used when a reinforcement-related neuron had been identified by its differential responses in the standard (VDS and/ or VDC) visual discrimination tasks.

The responses of one neuron (\#253) recorded in the SI during the performance of the reversal task is shown in Figure 1. In this particular experiment, the Red $\mathrm{S}+$ was presented first and lick responses to it resulted in juice delivery, while saline was delivered for responses to the Blue $S-$. Differential neuronal activity occurs during the first presentations of the 2 stimuli, in that there was an increase in firing rate to the Red $\mathbf{S}+$ and a decrease to the Blue $S-$. The first reversal trial occurred when a response to the Red stimulus ( $\operatorname{Red} \mathrm{S}-$ ) resulted in the delivery of saline. The neuron responded on this trial with an increase in firing before the delivery of saline, but the neuronal response to this stimulus then declined for subsequent presentations of the Red $S-$. In the case of the Blue $S+$, the neuron responds more on the first presentation of this stimulus after reversal (i.e., when responses to the red stimulus resulted in saline delivery) than when the Blue stimulus signaled saline, even though the monkey had not yet obtained juice through licking in response to this stimulus. Note that the neuronal response to the Blue $\mathrm{S}+$ on trial 9 occurs without a lick response, providing one type of evidence that the neuronal activity was not due to motor responses.

For each neuron, an analysis of variance was computed on the responses to the 2 stimuli during acquisition of the first contingency $($ Red $+/$ Blue - or Blue $+/$ Red - ) and after reversal. The majority of neurons showed significant differential responses during acquisition of the visual discrimination (SI: 12/ 14 neurons; DBB: 5/7 neurons). Significant differential responses which depended on the new reinforcement contingency were also found during the subsequent reversal of the discrimination (SI: 6/13 neurons; DBB: 4/6 neurons). Two neurons were tested during acquisition but not reversal. Although not all tests yielded statistically significant results, the direction of the neuronal responses always changed appropriate to the changed reinforcement contingency after reversal. Thus, the response to a particular stimulus depends upon the current reinforcement value of the stimulus learned by the monkey: for $S+$ neurons there is a response to the stimulus when it signals the availability of juice and a decrease in firing rate when the stimulus signals the availability of saline. The converse is true for $S-$ neurons. Moreover, the neuronal responses reverse in 1 or 2 trials.

\section{The recognition memory tasks}

The differential neuronal responses during the performance of the visual discrimination and reversal tasks indicate that learning influences the responses of basal forebrain neurons. In order 

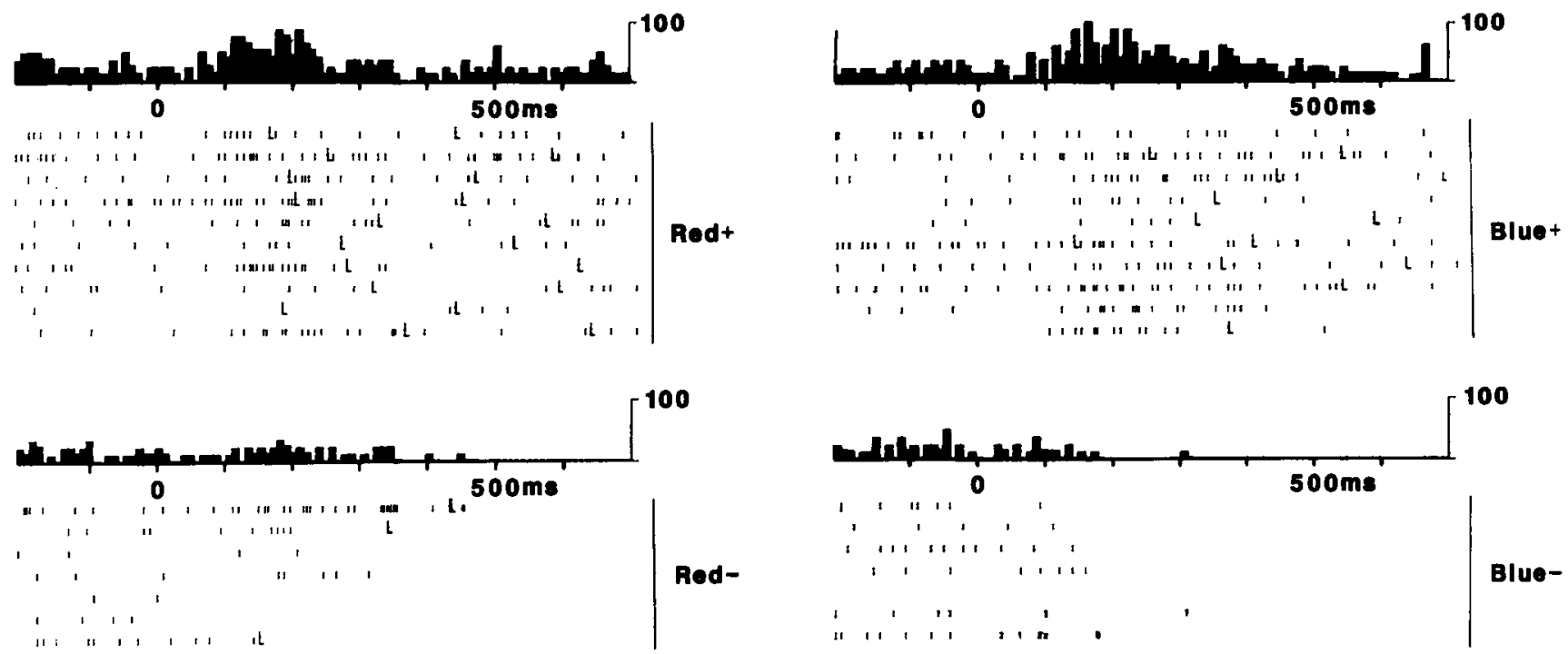

Figure 1. Responses of neuron \#253 in the visual discrimination reversal task. Each row represents the neuronal response to a stimulus (presented at time 0 ) on a single trial. The 2 discriminative stimuli were presented in pseudorandom order, but trials are grouped for clarity. The first stimulus presented was the Red $S+$, interdigitated with presentations of the Blue $S-$. The neuron responded appropriately to these stimuli, even though they were not seen for $18 \mathrm{hr}$. After 17 trials, the contingency was reversed (Red S-/Blue S+). Following the first trial in which saline was delivered in response to the former $\mathrm{S}+$, the neuron stops responding to this stimulus. Conversely, the response to the former $\mathrm{S}-$ is completely reversed after experience with the Red $\mathbf{S}-$. Note that the neuronal response to the Blue $\mathbf{S}+$ on trial 9 is not accompanied by a lick, thus dissociating the neuronal response on the basis of the reinforcement value of the stimulus from the motor response. There is a biphasic response to the presentation of these stimuli: an increase in firing rate for the $\mathrm{S}+$ and a decrease for the $\mathrm{S}-$. The $L$ indicates the occurrence of the lick response. The scale at the right of the histogram represents a firing rate of $100 \mathrm{spikes} / \mathrm{sec}$. Bin width $=10 \mathrm{msec}$. See also Figure 2 .

to study the effect of learning and memory further, differential neurons tested in the visual discrimination tasks were also tested during performance of recognition memory tasks in which each stimulus was presented twice a day, initially as novel and then as familiar. Lick responses to a novel stimulus resulted in the delivery of saline, while responses to the same stimulus when familiar resulted in the delivery of fruit juice.

It was found that reinforcement-related neurons responded to the stimuli in the recognition memory tasks on the basis of their reinforcement value, as in the visual discrimination tasks. Examples of the responses of these neurons during the performance of the recognition memory task are shown in Figure 2. For comparison, the responses of this neuron are shown during the performance of the reversal task (Fig. 1).

In the case of $S+$ neurons, the response to familiar stimuli (and the $\mathrm{S}+$ ) was an increase in firing rate with a different response to novel stimuli (and the $S-$; e.g., neuron \#253, Figs. $1,2)$. For $S-$ neurons, the main response was an increase in firing rate to novel stimuli and the $\mathbf{S}-$ (e.g., Figs. 6, 7). This pattern of responses was seen in all 3 regions of the basal forebrain.

The scatter plots in Figure 3 show the data for all neurons tested in the RMC (computer-based recognition memory) task. Each data point represents the response of an individual neuron to novel and familiar stimuli, plotted along the abscissa and ordinate, respectively. The spontaneous firing rate of a neuron was subtracted from the stimulus-elicited responses, each data point thus representing the magnitude and direction of the change in the neuronal responses produced by the stimuli. The responses of $S+$ neurons cluster around the ordinate, while the responses of $S-$ neurons cluster around the abscissa. Some neurons show a biphasic pattern of response, e.g., an increase in firing rate to familiar stimuli and a decrease to novel stimuli. The intercepts and slopes of regression lines calculated on the responses of the differential neurons are similar, indicating that the reinforcement-related neuronal populations in the 3 regions of the basal forebrain respond similarly.
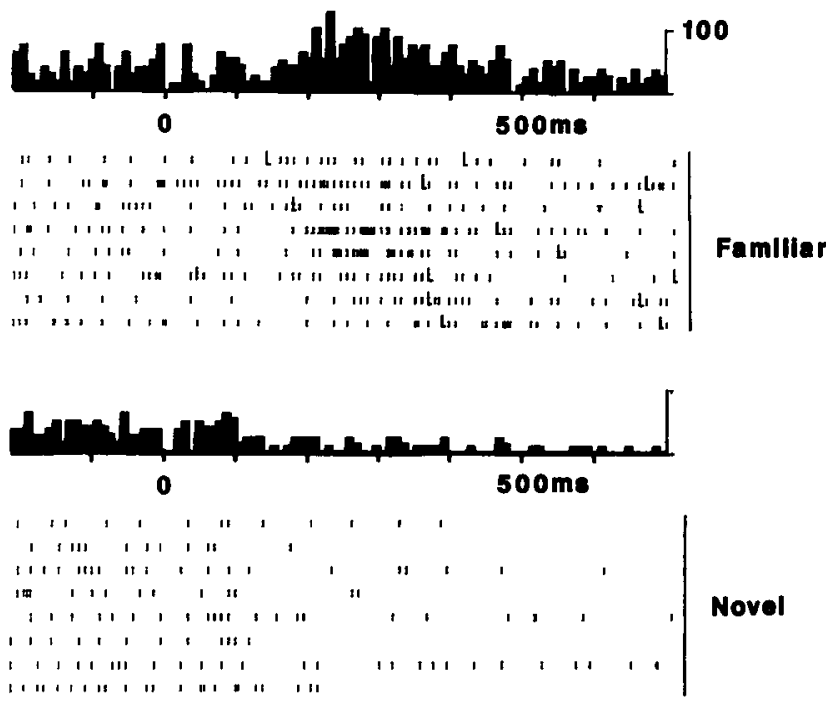

Figure 2. Responses of a reinforcement-related neuron (\#253) in the recognition memory task. Presentations of novel and familiar stimuli (onset at time 0 ) occurred in pseudorandom order but are grouped for clarity. The neuron (see also Fig. 1) responds with an increase in firing to familiar stimuli and a decrease to novel stimuli. The $L$ indicates the occurrence of the lick response. The scale at the right of the histogram represents a firing rate of 100 spikes $/ \mathrm{sec}$. Bin width $=10 \mathrm{msec}$. 

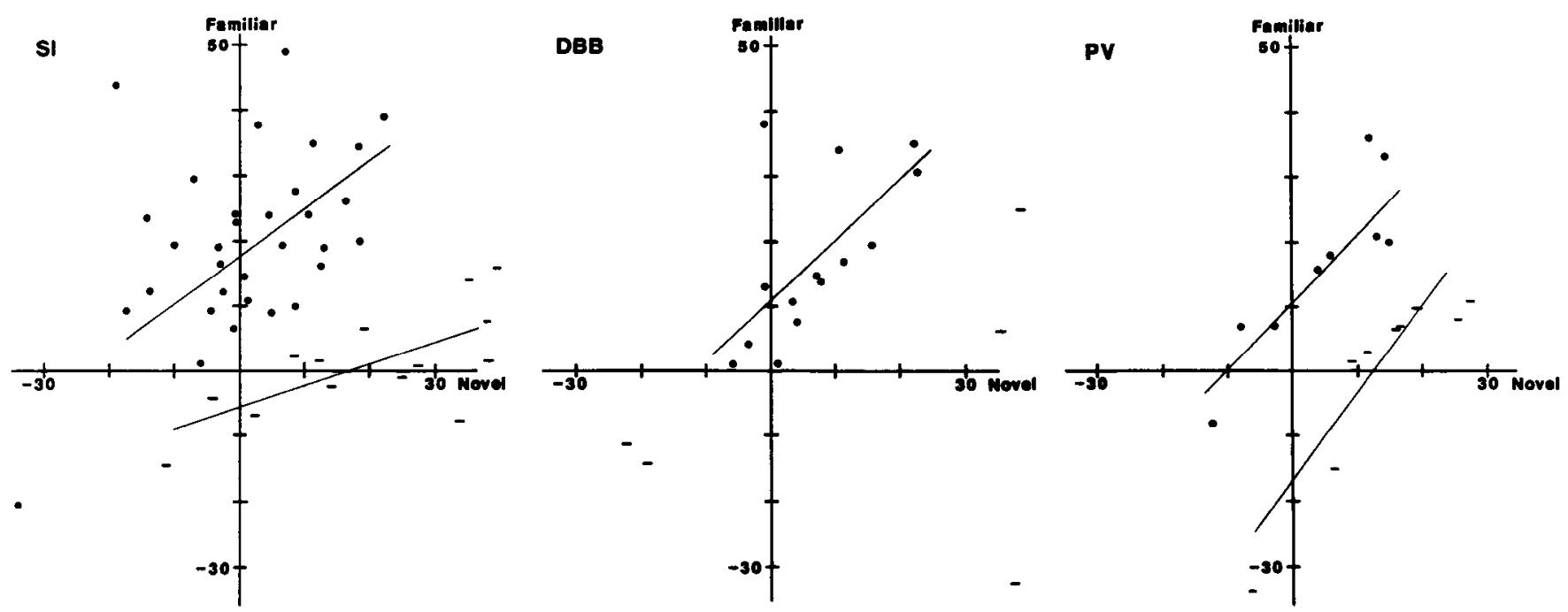

Figure 3. Comparison of the responses to novel and familiar stimuli for the 3 basal forebrain regions. Each data point represents the mean responses (spikes/sec) of one neuron to familiar (ordinate) and novel stimuli (abscissa) from which the spontaneous firing rate of that neuron was subtracted. This transformation demonstrates the magnitude and direction of the neuronal responses. Neurons are grouped into $S+$ type (filled circles) and $S$ - type (-). Many neurons show a biphasic response to the stimuli, e.g., an increase in firing rate to familiar stimuli and a decrease in firing rate to novel stimuli. Regression lines represent different groups of neurons for which significant correlations between the responses to novel and familiar stimuli were obtained: SI ( $S+$ neurons: $y=19.7 x+0.5, r=0.46, p<0.01 ; S-$ neurons: $y=-5.6 x+0.3, r=0.67 ; p<0.01)$; DBB: $(S+$ neurons: $y=11 x \mid 0.9, r=0.64, p<0.05 ; S$ neurons: not significant); and PV region $(S+$ neurons: $y=10.3 x+1.13, r=0.79, p$ $<0.05 ; S-$ neurons: $y=-16.9 x+1.3, r=0.9, p<0.01)$.

The responses to novel and familiar stimuli were invariably in the same direction as the responses to the $\mathbf{S}-$ and $\mathbf{S}+$, although the magnitude of the responses were often not as great as to the stimuli used in the visual discrimination task. Statistical analysis confirmed that the majority of neurons in the SI and $\mathrm{PV}$, tested in one or both of the recognition memory tasks, showed significantly different responses in the recognition memory task. Fewer neurons showed significant differences in the DBB, although the direction of the responses to novel and fa- miliar stimuli was appropriate to the reinforcement contingency (Table 1).

As most neurons tested in the recognition tasks had been tested in visual discrimination tasks (Wilson and Rolls, 1989a), the analysis of variance and Tukey tests were used to compare the responses to the stimuli in the recognition task with those in the discrimination task. This analysis showed that the majority of neurons showed significant differences between the $\mathrm{S}+$ and novel stimuli, and between the $S-$ and familiar stimuli:

\begin{tabular}{|c|c|c|c|}
\hline & SI & DBB & PV \\
\hline \multicolumn{4}{|l|}{ A } \\
\hline No. of differential units & 73 & 24 & 23 \\
\hline \multicolumn{4}{|l|}{ B } \\
\hline \multicolumn{4}{|l|}{ Neurons tested in: } \\
\hline VD tasks & 67 & 24 & 14 \\
\hline RM tasks & 46 & 19 & 17 \\
\hline RM only & 6 & 0 & 9 \\
\hline \multicolumn{4}{|l|}{$\mathrm{C}$} \\
\hline \multicolumn{4}{|l|}{ Recognition tasks } \\
\hline \multicolumn{4}{|c|}{ No. of significant tests/neurons tested: } \\
\hline RMC task (Computer) & $25 / 33(76 \%)$ & $5 / 13(38 \%)$ & $11 / 15(73 \%)$ \\
\hline RMS task (Shutter) & $21 / 29(72 \%)$ & $3 / 12(25 \%)$ & $4 / 5(80 \%)$ \\
\hline \multicolumn{4}{|l|}{ Discrimination Tasks } \\
\hline \multicolumn{4}{|c|}{ No. of significant tests/neurons tested: } \\
\hline VDC task (Computer) & $34 / 41(82 \%)$ & $14 / 18(78 \%)$ & $8 / 8(100 \%)$ \\
\hline VDS task (Shutter) & $40 / 42(95 \%)$ & $15 / 15(100 \%)$ & $4 / 4(100 \%)$ \\
\hline
\end{tabular}




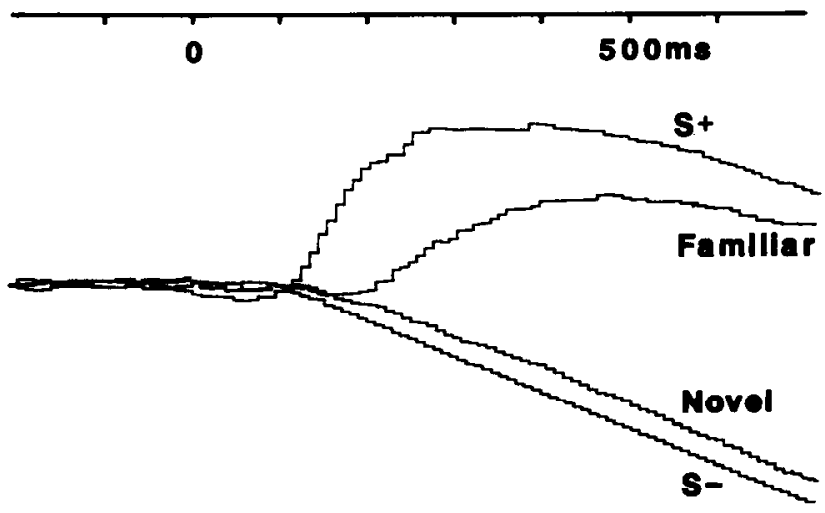

Figure 4. Analysis of differential response latencies using cumulative sum histograms (neuron \#253). Each histogram represents the neuronal responses on one of the different types of trial. The data were obtained from the responses of neuron $\# 253$ in the recognition memory (Fig. 2). Responses to the $\mathbf{S}+$ and familiar stimuli are increases in firing rate; the response to familiar stimuli follows that to the $S+$ by $100 \mathrm{msec}$. Responses to novel stimuli and the $S-$ are opposite to the other stimuli, i.e., decreases in firing rate.

overall, 72,63 , and $75 \%$ for the SI, and DBB, and the PV region, respectively (Table 2 ). These differences are consistent with the differential reinforcement signaled by the stimuli.

\section{Differential response latencies}

During the performance of the recognition memory task, the monkey saw each stimulus only twice a day. However, differential neurons responded to novel stimuli in the same way as they did to the $\mathrm{S}-$, despite the fact that the monkey was looking at the stimulus for the first time. This indicated that the reinforcement value of a novel stimulus is encoded in the responses of these neurons during the first presentation of a stimulus.

The time taken for a neuron to respond differentially to novel compared to familiar stimuli is termed the differential response latency. This was determined by constructing cumulative sum histograms (see Materials and Methods) and measuring the latency of the inflection point indicating changes in response to the stimuli. The change in the slope of the histogram represents the change in firing rate for a particular type of trial. The cumulative sum histograms of neuron \#253 (Fig. 4) show that the responses to novel and familiar stimuli are different in their direction (increases in firing to familiar stimuli compared with decreases in firing to novel stimuli). For comparison, histograms of the responses of this neuron to the $S+$ and $S-$ are also shown in Figure 4. The responses to the $\mathrm{S}+$ and familiar stimuli are similar (as are the histograms for responses to novel stimuli and

Table 2. Results of the analyses of variance, comparing responses to $\mathbf{S}+$ with novel stimuli and $\mathbf{S}-$ with familiar stimuli

\begin{tabular}{llll} 
Comparison & $\begin{array}{l}\text { Substantia } \\
\text { innominata }\end{array}$ & $\begin{array}{l}\text { Diagonal } \\
\text { band } \\
\text { of Broca }\end{array}$ & $\begin{array}{l}\text { Periven- } \\
\text { tricular } \\
\text { region }\end{array}$ \\
\hline $\begin{array}{l}\mathrm{S}+/ \text { novel } \\
\mathrm{S}-/ \text { familiar }\end{array}$ & $26 / 40(65 \%)$ & $13 / 19(68 \%)$ & $6 / 8(75 \%)$ \\
\hline
\end{tabular}

Percentage values represent the number of significant differences for each group.
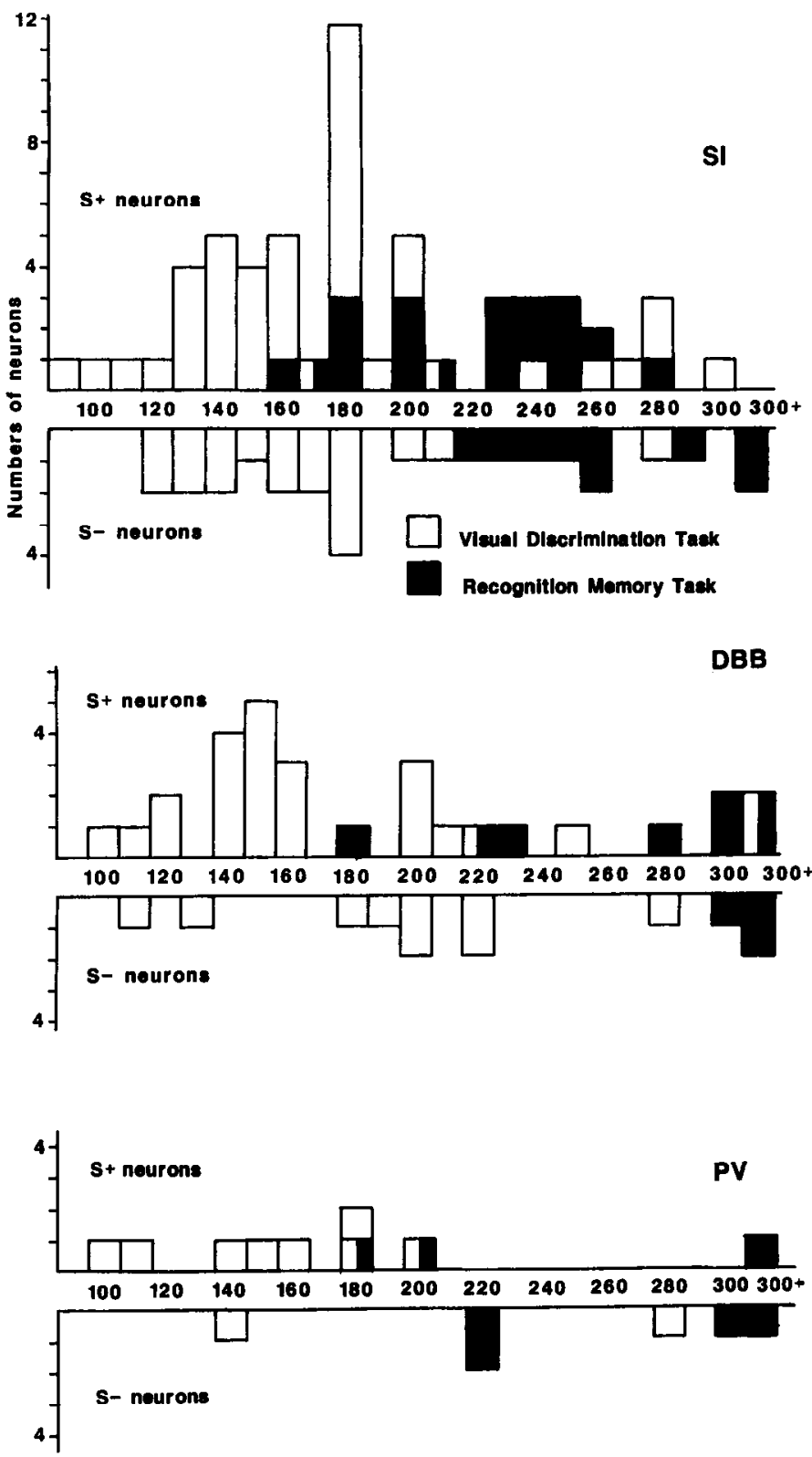

Figure 5. Distribution of differential response latencies for reinforcement-related neurons in the three regions of the basal forebrain. Latencies for $S+$ type and $S$ - type neurons are shown above and bclow the horizontal axes, respectively; the 2 distributions are very similar. The shaded histograms represent data for the recognition memory task, while values for the visual discrimination task are unshaded. The latencies for the recognition tasks are notably longer than in the discrimination task.

the $\mathrm{S}-$ ), except that the response to the $\mathrm{S}+$ precedes in time the response to familiar stimuli. In fact, the differential response latencies of all neurons determined in the visual discrimination task preceded the differential response latency for the recognition memory task. The mean differential response latencies for the recognition memory and visual discrimination tasks for the 3 basal forebrain regions are shown in Table 3. Figure 5 shows the distribution of differential response latencies for the 2 tasks in the 3 regions, which are similar. It is notable that the latencies of $S+$ and $S-$ neurons are also similar. 


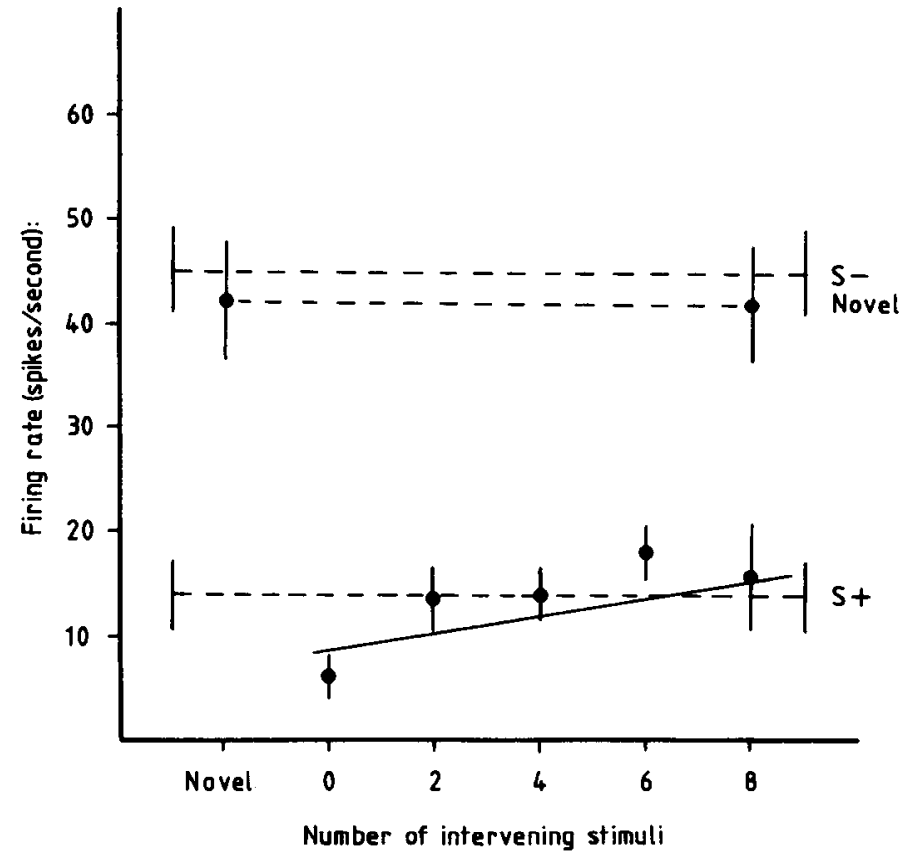

Figure 6. Effect of intervening trials on the differential responses to novel and familiar stimuli. Neuron $\# 51$ responds maximally to the $\mathbf{S}-$ and novel stimuli. The neuron was tested on the recognition memory task with up to 8 intervening trials between presentations of novel and familiar stimuli. Data points represent the mean (and SEM) response to familiar stimuli for a particular number of intervening trials. The response to familiar stimuli presented on the trial immediately $(8 \mathrm{sec})$ following the novel presentation is a decrease in firing rate below spontaneous firing rate ( $12 \mathrm{spikes} / \mathrm{sec})$. There is a significant increase in firing rate with increasing numbers of intervening trials $(r=0.38 ; p<0.01)$. A linear regression indicates that the decrement in response to familiar stimuli decays to zero (to that of the response to novel stimuli) after 36 intervening trials.

\section{Effect of intervening trials on the magnitude of the differential response}

The recognition memory task allows stimuli to be presented that are novel or, if they are familiar, have not been seen by the monkey for a number of trials. This task allows the testing of memory for the stimuli in the presence of interference generated by other stimuli. For 42 neurons, a comparison was made between the neuronal responses to novel stimuli and the subsequent responses to familiar stimuli seen after a number of other intervening trials. This comparison was effected by computing the Pearson product-moment correlation to test for changes in the neuronal response to familiar stimuli due to the effect of intervening trials or due to the passage of time and thus allows the determination of the "memory span" of the neuron.

Figure 6 represents data from one neuron showing the responses to novel stimuli and responses to presentations of these stimuli when shown as familiar. These mean values are plotted as a function of the number of intervening trials since the first, novel presentation of the stimuli. The data for this experiment were drawn from the presentations of 89 novel stimuli and their subsequent familiar presentations. Despite the large number of data points, the correlation coefficient is low, although significant $(r=0.38, p<0.01)$. The slope of the regression line is shallow as the responses to familiar stimuli did not vary greatly as a function of the number of intervening trials between the 2
Table 3. Analysis of differential response latencies obtained in visual discrimination (VD) and recognition memory (RM) tasks

\begin{tabular}{llll} 
& \multicolumn{3}{c}{ Differential response latency (msec) } \\
\cline { 2 - 4 } Task & $\begin{array}{l}\text { Substantia } \\
\text { innominata }\end{array}$ & $\begin{array}{l}\text { Diagonal } \\
\text { band } \\
\text { of Broca }\end{array}$ & $\begin{array}{l}\text { Periven- } \\
\text { tricular } \\
\text { region }\end{array}$ \\
\hline VDC task & 175 & 153 & 168 \\
S+ type neurons & 160 & 200 & 170 \\
S- type neurons & 175 & 203 & 105 \\
VDS task & 171 & 176 & 280 \\
S+ type neurons & & & \\
S- type neurons & 223 & 315 & 200 \\
RMC task & 282 & 410 & 270 \\
S+ type neurons & & & \\
S- type neurons & 243 & 240 & 180 \\
RMS task & 242 & 470 & 260 \\
S+ type neurons & & & \\
S- type neurons & & & \\
\hline
\end{tabular}

The data are separated into tasks in which stimuli are generated by computer (VDC; RMC) on a video monitor or using the shutter (VDS; RMS). Mean values are in milliseconds after the onset of the stimulus presentation.

presentations of the stimuli. This effect suggests that the memory for the stimuli influences the neuronal response even when the stimuli had not been seen for a number of intervening trials. For most neurons, which were similar to this example, differences in the responses to familiar stimuli due to intervening trials were small, and most of the correlation coefficients were not significant.

In order to estimate the "memory spans" of the neurons, linear regressions were calculated on firing rate as a function of the number of intervening stimuli. In most cases, the slopes were very shallow, as shown in Figure 6. The regression lines were used to estimate the decay of the differential neuronal response, to determine the point at which the response to familiar stimuli was equal to the response to novel stimuli, i.e., the "memory span" of the neuron. The estimated "memory spans" for the 3 basal forebrain structures were as follows: substantia innominata, mean $=36$ trials; range $=2-82$ trials; diagonal band, mean $=31$ trials; range $=14-51$ trials; periventricular region, mean $=21$ trials; range $=5-71$ trials. In 16 cases, the direction of the regression slope indicated that the memory span did not decline or increased with large numbers of intervening trials. The memory span values of these neurons were so long that it was not possible to estimate them. Overall, these data show that differential basal forebrain neurons will respond appropriately to reinforcing stimuli even when not seen for many trials provided that the monkey has correctly determined the reinforcement value of a familiar stimulus.

\section{Dissociations between eye movements, lick movements, and neuronal responses}

Eye movements and differential neuronal responses do not occur at the same time. Eye movements occurring when the monkeys fixate novel and familiar stimuli during the recognition memory tasks are small $\left(<1^{\circ}\right)$ and are essentially identical on both types of trial during the $500 \mathrm{msec}$ period in which neuronal activity was collected (Fig. 7, $A, B$ ). This can be compared with the differential neuronal responses to novel and familiar stimuli which occur while fixation is largely constant. The largest eye 

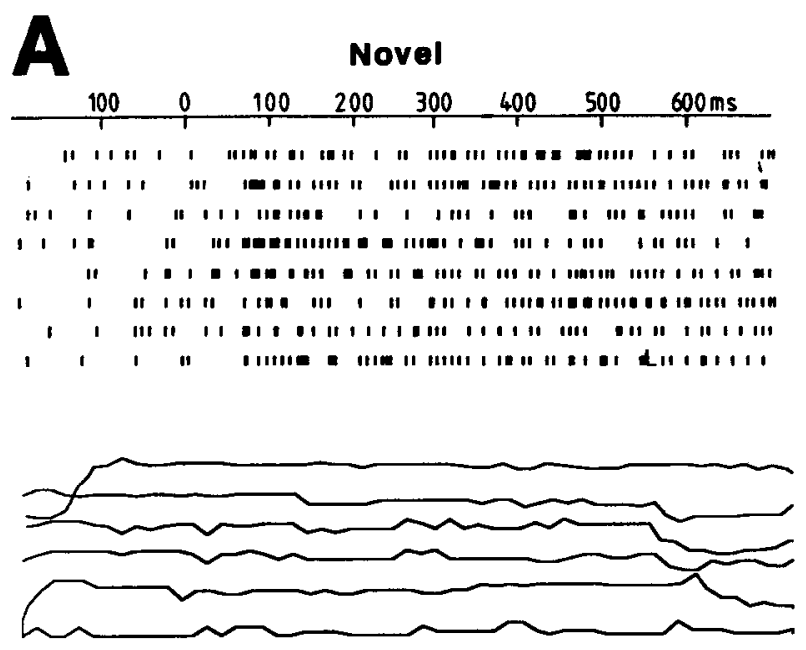

B

\section{Famillar}

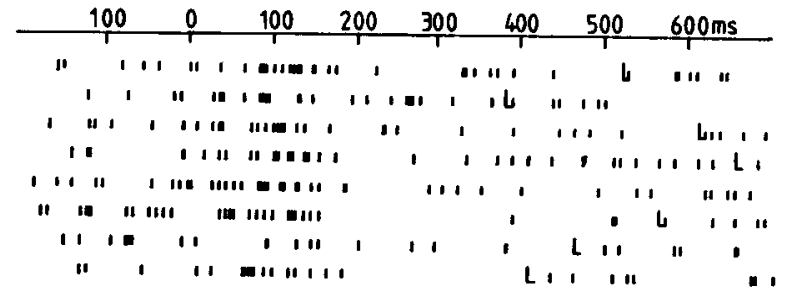

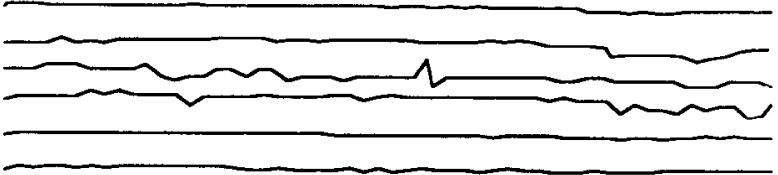

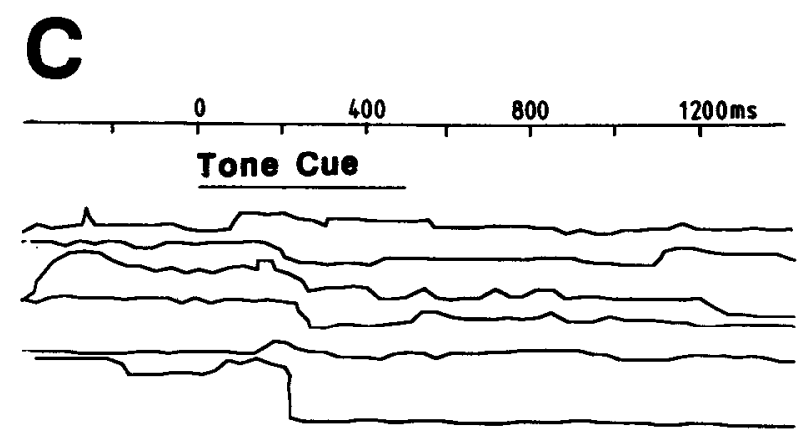

Figure 7. Comparison of horizontal eye movements and differential neuronal responses. Neuron \#45 responds differentially to novel $(A)$ and familiar $(B)$ stimuli at a latency of $200 \mathrm{msec}$, while eye movements are negligible at this time, and similar on both novel and familiar trials. The largest eye movements $\left(5^{\circ}\right)$ occur during the tone cue period $(C)$ and after the behavioral responses have occurred. The EOG records were taken from the first 6 trials shown in the raster displays on novel and familiar trials. Note that the monkey erroneously licks late on novel trial 8 and fails to lick on familiar trial 5 , although the neuron responds appropriately to the stimuli, thus dissociating the reinforcement-related neuronal responses and the behavioral responses.

movements observed usually followed the presentation of the tone cue (Fig. 7C), during which the monkeys' eyes moved to fixate the shutter or the video monitor where the visual stimuli wcrc to appcar. In Figure $7 A$, it is noteworthy that an erroneous lick response occurs late on trial 8 in which a novel stimulus was presented. Lick responses were usually made at the presentation of familiar stimuli when the neuron responded with a decrease in firing rate, yet the erroneous lick occurred when the neuron responded with an increase in firing rate to the novel stimulus. Conversely, on trial 5 (Fig. $7 B$ ) the neuron responds appropriately with respect to the reinforcement contingency, even though the monkey does not make a lick response. Thus, the neuronal activity reflects the reinforcement contingency rather than the behavioral responses.

\section{Anatomical location of the reinforcement-related neurons}

As described in Materials and Methods, the locations of the reinforcement-related neurons were determined based on the positions of microlesions and $\mathrm{X}$-radiographs of the microelectrodes in situ. Figure 8, $A, B$, illustrates the location of microlesions made at the site of the most anterior recording in the DBB and at the site of a reinforcement-related neuron located at the dorsal margin of the anterior commissure and putamen. Figure
$8 C$ shows the locations of all reinforcement-related neurons in the SI, the DBB, and the periventricular region. These reconstructed locations have been plotted on standard series of sections every $2 \mathrm{~mm}$ relative to the interaural line. The positions of these neurons are often in conjunction with fiber tracts such as the anterior commissure, internal capsule, inferior thalamic peduncle, and ansa peduncularis. The cell bodies of the basal nucleus of Meynert are also distributed adjacent to these fiber bundles, and several studies (Parent and Debellefeuille, 1982; Mesulam et al., 1983; Hedreen et al., 1984) have observed interstitial cells of the basal nucleus at the borders of the anterior commissure, putamen, and pallidum, as shown in Figure $8 B$. It is possible that the reinforcement-related neurons are part of the basal nucleus of Meynert.

\section{Discussion}

The experiments described in this paper indicate that neurons recorded in 3 regions of the basal forebrain encode the learned reinforcement value of visual stimuli, irrespective of the sensory properties of the stimuli signaling reinforcement or the different reinforcement contingencies that had to be learned in order to perform the visual discrimination reversal and recognition memory tasks. The evidence for this is that reinforcement-re- 

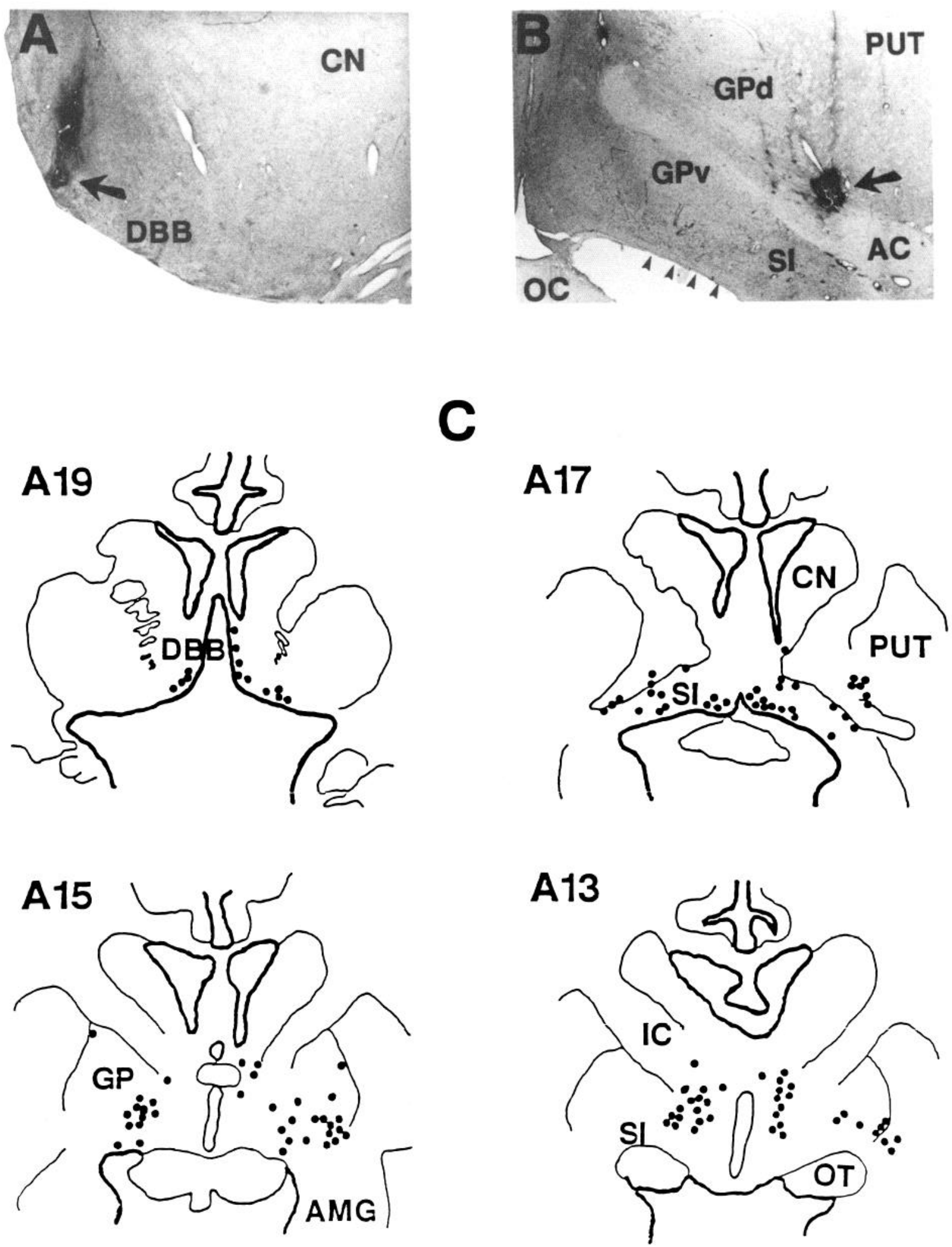

Figure 8. Locations of reinforcement-related neurons. $A$ and $B$, Photomicrographs of brain sections in which lesions (large arrows) were made during electrode tracks into the basal forebrain. The small arrows indicate the proximity of cells of the basal nucleus of Meynert to the base of the brain. The recordings often traversed this region, as indicated by the location of reinforcement-related neurons shown in $C$. The drawings in $C$ are representative sections at 4 different levels though the basal forebrain. Each dot represents the reconstructed location of a single neuron with reinforcement-related activity. Abbreviations: $A C$, anterior commissure; $A M G$, amygdala; $C N$, caudate nucleus; $D B B$, diagonal band of Broca; $G P$ ( $d$ or $v$ ), globus pallidus (dorsal or ventral); $I C$, internal capsule; $O T$, optic tract; $P U T$, putamen; $S I$, substantia innominata. 
lated neurons responded appropriately to changes in reinforcement contingency during the acquisition and reversal of a visual discrimination task and that the same neurons also responded differentially in 2 versions of a recognition memory task, in which a single presentation of a given stimulus was sufficient to elicit a differential response. These differential responses reflected the reinforcement value of the stimuli, indicating that prior recognition of the novelty and familiarity of the visual stimuli and access to a durable memory store had occurred, in order to produce the neuronal responses. However, the basis of the neuronal response was the reinforcement value of the stimuli, for although the neurons responded differentially to novel and familiar stimuli in the recognition task, they responded differentially to the $\mathbf{S}+$ and $\mathbf{S}$ - in the reversal task, stimuli that were equally familiar, but differentially reinforcing.

The responses of neurons with reinforcement-related activity are not related to arousal and movements caused by manipulation of the trunk and limbs or by the ingestion of aversive saline (Rolls et al., 1976; Wilson and Rolls, 1989a). The responses of these neurons in the recognition memory task also support this contention. Behavioral studies have shown that monkeys find novel stimuli arousing and that such stimuli elicit approach behavior (Humphrey, 1972; Mishkin and Delacour, 1975). In the present experiment, the monkeys had to avoid making responses to novel stimuli, responding to them only when they were familiar. Yet it is likely that these stimuli were arousing, for the monkeys showed excellent memory for the stimuli in the recognition task, with accurate responses to them, both when novel and when familiar. Thus, while all the stimuli used in the tasks were arousing for the monkeys, the neurons responded differentially to them, depending upon their current reinforcement value.

There are several ways in which the reinforcement-related neuronal activity can be dissociated from movements. First, neuronal responses could occur to stimuli signaling juice when the monkey failed to make a behavioral response (Fig. 1). Second, the monkey could make an erroneous lick response on a trial in which a novel stimulus was presented, yet the neurons responded appropriately to the contingency (Fig. 7). Third, neuronal responses did not accompany erroneous lick movements occurring in the intertrial interval. Finally, eye movements do not account for the differential neuronal activity (Fig. 7).

Thcse rcsults arc of interest because neuropsychological studies have implicated the basal forebrain in cognitive processes. The data in this paper clearly show that reinforcement-related neuronal responses reflect the learning of complex contingencies, behavioral flexibility, and access to memory for visual stimuli. These results are consistent with the finding that lesions of the basal forebrain impair the performance of visual discrimination and object recognition tasks (Aigner et al., 1984; Ridley et al., 1985, 1986). The means by which basal forebrain neurons gain access to this information and the implications for cognitive function and the functional role of the basal forebrain are discussed below.

\section{Responses of basal forebrain neurons are related to learning and memory}

Physiological and neuropsychological studies have indicated that the basal forebrain contributes to the control of both motivated behavior and cognitive processes. The responses of basal forebrain neurons are indicative of a coupling of these processes. Individual basal forebrain neurons participate in at least 2 forms of motivated behavior, feeding and drinking, their activity reflecting the current motivational objectives of the monkey responding to stimuli for which the monkey is prepared to work (Wilson and Rolls, 1989a). Their activity also reflects a state in which the identity and motivational significance of a stimulus has been determined, indicating access to information about a stimulus and its properties, and the learning of reinforcement contingencies. These neuronal responses thus reflect the expected availability of or opportunity for reinforcement.

The differential neuronal responses are clearly the results of learning. In order to perform a visual discrimination task, the monkey has to learn to respond differentially to 2 stimuli. Our experiments required learning of 2 discrimination tasks, one discrimination reversal and 2 recognition memory tasks. Individual neurons tested in these different tasks consistently responded on the basis of the learned reinforcement value of the stimuli in these tasks, despite the different contingencies required for performance. The differential responses to the many stimuli utilized in these tasks reflected their reinforcement value and show that these neurons are active not simply in relation to the sensory properties of the stimuli.

The visual discrimination reversal and recognition memory tasks both require reversals of the behavioral responses. In both tasks, the contingencies change so that the response to a stimulus on one trial may be inappropriate on a subsequent trial. Differential neurons clearly follow the change in the contingency, and as the monkey learns that the reversal contingency has taken place, the neuronal responses also change.

The rate of learning reflected in the neuronal activity appears to be very rapid in well-trained monkeys. In a visual discrimination reversal task, the differential activity of reinforcementrelated neurons appeared on the first acquisition trials on which the stimuli were presented, even when the stimuli had not been seen for $18 \mathrm{hr}$. Further, the neuronal responses changed within 3 trials when the reinforcement value of a stimulus was reversed, the neuronal changes occurring as quickly as the change in the monkey's behavior. In the recognition memory tasks, a single trial is sufficient to result in differential activity as the responses to novel stimuli are similar to those to the $S-$, and the responses to familiar stimuli are similar to the responses to the $\mathbf{S}+$. Indeed, the analysis of the differential response latencies shows that the novelty/familiarity of the stimulus is reflected in the neuronal response within $250 \mathrm{msec}$ on average. It is of interest that the discrimination latencies obtained in the recognition memory task were generally $70-100 \mathrm{msec}$ longer than in the visual discrimination task, presumably reflecting the additional processing necessary to determine stimulus novelty/familiarity and the appropriate reinforcement value of the stimuli.

The differential neuronal responses reflect a durable memory for the reinforcement value of visual stimuli. In the recognition memory task, the novelty/familiarity of the stimulus is the cue that determines the reinforcement value of the stimulus. The responses of these differential neurons clearly reflect access to information from memory about the novelty/familiarity of the stimuli, information that appears to be maintained in memory over many trials, as judged by the behavioral performance and the differential neuronal responses to familiar stimuli that had not been recently seen.

The data strongly support the idea that the neuronal responses reflect memory for reinforcement value. However, these neurons do not simply reflect direct associations between stimuli and reinforcers. In the recognition task, the differential response 
is apparent on the first trial that a novel stimulus is presented, and as the monkeys received saline on fewer than 1 in 10 novel trials, frequent delivery of saline reinforcement is not necessary to elicit differential responses once the contingency is learned. Further, differential responses to familiar stimuli occur before the monkey has received juice from a lick response to these stimuli, and so a direct association between the stimulus and a reinforcer cannot explain the neuronal responses to familiar stimuli. Thus, the learned rule that responses to novel and familiar stimuli are differentially reinforcing is apparently able to guide behavioral responses and is presumably responsible for the differential neuronal responses, indicative of the cognitive mechanisms underlying the responses of these basal forebrain neurons. These results confirm previous studies which have shown that basal forebrain neurons respond to stimuli that monkeys have learned are reinforcing (Travis and Sparks, 1969) and cease to respond to reinforcers during extinction, when the monkey has learned that reinforcement, signaled by a visual stimulus, is to be withheld (Mora et al., 1976).

\section{Do reinforcement-related neurons play a role in memory?}

The data obtained in the reversal and recognition tasks show that basal forebrain neurons have access to information about reinforcement contingencies and memory for the novelty and familiarity of visual stimuli, thus indicating that the basal forebrain is part of, or receives the output from, memory mechanisms.

In contrast to the basal forebrain, neurons responding to reinforcing stimuli in the orbitofrontal cortex and amygdala are more specific in the particular stimuli to which they respond, and such neurons in the amygdala do not respond differentially to novel and familiar stimuli when this information is important in obtaining reinforcement (Thorpe et al., 1983; Wilson and Rolls, 1985, 1987). The output of these afferent neurons appears to be combined in the basal forebrain, producing a neuronal signal that represents the expected availability of reinforcement rather than stimulus attributes such as shape, color, or familiarity.

If the reinforcement-related neuronal activity is to influence memory processes, anatomical connections must exist that allow the basal forebrain to facilitate the specific functional mechanisms of cortical ensembles, optimizing their functioning when appropriate, such as during the performance of cognitive tasks. There is abundant evidence that the basal forebrain projects to the cerebral cortex (e.g., Mesulam et al., 1983), and between 70 and $90 \%$ of neurons in the substantia innominata and vertical limb of the diagonal band of Broca are cholinergic. The present recordings took place in these structures and thus some of the recorded neurons are likely to be cholinergic. This possibility is strengthened by the finding that reinforcement-related neurons project to the cerebral cortex and thus are part of the basal nucleus of Meynert (Wilson et al., 1984; Rolls, 1986; Wilson and Rolls, 1989a).

The concentration of cholinergic markers in limbic cortical regions is particularly high (Mesulam et al., 1984, 1986). As limbic cortical regions are known to be important for memory function, it may be through this cholinergic innervation that the basal forebrain affects memory function. Reinforcement-related neurons respond to salient cues that the monkey has learned are important and thus could facilitate memory processes, and the utilization of information already in store (Rolls, 1987). This role is consistent with studies showing that changes in brain
ACh influence memory in man (Drachman, 1977) and in monkey (Dean and Bartus, 1985; Aigner et al., 1987), and that large lesions of the basal forebrain impair the learning and performance of visual discrimination and object recognition tasks (Aigner et al., 1984; Ridley et al., 1985, 1986). It has also been shown that lesions of the basal forebrain significantly decrease the proportions of cortical neurons that respond to an auditory cue that signaled the delivery of reinforcing brain stimulation (Rigdon and Pirch, 1986). Finally, mechanisms that may play a specific role in memory by encoding the novelty and familiarity of visual stimuli are also known to exist in the basal forcbrain (Rolls et al., 1982; Wilson, 1989; Wilson and Rolls, 1989b).

\section{The importance of cortical afferents for the basal forebrain}

The responses of basal forebrain neurons reflect learning about the complex relationships between stimuli in several modalities and the contingencies learned by the monkeys. It is possible that these mechanisms exist within the neuronal circuitry of the basal forebrain or, alternatively, are dependent upon afferent structures. A review of the functional role and connections of these afferent regions (Wilson et al., 1988; Wilson and Rolls, $1989 a, b)$ strongly suggests that the properties of basal forebrain neurons are derived from inputs from these structures, especially limbic cortical regions.

The cortical regions projecting to the basal forebrain are few, limited to the ventromedial regions of the prefrontal and temporal cortices, the cingulate cortex, and insula (Whitlock and Nauta, 1956; Jurgens and Muller-Preuss, 1977; Leichnetz and Astruc, 1977; Mesulam and Mufson, 1984; Russchen et al., 1985); the subcortical inputs include the amygdala, the thalamus, supramammillary region, and midbrain (Jones et al., 1976; Aggleton and Mishkin, 1984). The ventromedial regions of the prefrontal and temporal cortices are distinct in being the final cortical projection sites at the endpoint of a sequence of pathways originating in the visual cortex (Jones and Powell, 1970), which could provide the basis for the neuronal responses to visual stimuli in the basal forebrain. It is worth noting that performance of the recognition memory task depends upon determination of the identity of the stimuli utilizing information about shape, a function dependent upon the temporal cortex (Mishkin, 1982). The anatomical connections between the temporal and prefrontal cortex and the basal forebrain may underly the responses of basal forebrain neurons to visual stimuli, and the proximity of some reinforcement-related neurons to fiber tracts such as the anterior commissure, internal capsule, inferior thalamic peduncle, and ansa peduncularis (Fig. 8) may reflect possible functional connections with temporal and prefrontal structures.

The cortical regions projecting to the basal forebrain are known to play a role in memory function. It has been known for many years that damage to the ventromedial temporal and prefrontal cortical regions produces profound deficits in memory function in man and monkey (Scoville and Milner, 1957; Luria et al. 1967; Talland et al., 1967; Mishkin, 1978; Wallesch et al., 1983; Zola-Morgan and Squire, 1985; Bachevalier and Mishkin, 1986). The subcortical regions projecting to the basal forebrain also play a role in memory function (Mair et al., 1979; Squire and Moore, 1979). These observations are consistent with the hypothesis that the responses of basal forebrain neurons reflect, at least in part, cortical inputs.

Furthermore, damage to the basal forebrain in man produces a profound amnesia and even dementia when this occurs in 
conjunction with injury to the ventromedial prefrontal and temporal cortical regions (Friedman and Allen, 1969; Gascon and Gilles, 1973; Damasio et al., 1985a, b). It is not clear what the effect of a selective lesion has when confined to the basal forebrain in man. However, such lesions in monkeys produce impairments in a recognition memory task (Aigner et al., 1984) requiring judgments of familiarity similar to that of the present study, indicating that the basal forebrain contributes to memory function. An alternative hypothesis is that basal forebrain lesions disconnect memory mechanisms from their functional outputs.

A striking feature of the responses of reinforcement-related basal forebrain neurons is that they reflect robust memory for the novelty/familiarity of the stimuli presented in the recognition memory task. Thus, it is possible that these neuronal responses, which reflect access to recognition memory, are mediated by the cortical regions that provide their afferent input. This is consistent with the finding that damage or cooling of ventromedial temporal and prefrontal cortices impairs the performance of object recognition tasks in monkeys (Voytko, 1985; Bachevalier and Mishkin, 1986; Murray and Mishkin, 1986; Horel et al., 1987) and that these cortical regions project directly to the basal forebrain (Jurgens and Muller-Preuss, 1977; Mesulam and Mufson, 1984; Room et al., 1985; Russchen et al., 1985; Wilson, 1989; Wilson and Rolls 1989b). Furthermore, neurons that respond on the basis of stimulus novelty have been recorded in the ventromedial temporal cortex (Brown et al., 1987; Wilson et al., 1988) and may provide the memory-related information reflected in the responses of basal forebrain neurons.

\section{The basal forebrain, the cerebral cortex, and Alzheimer's disease}

Degeneration of the basal forebrain may be responsible for the memory impairments in Alzheimer's disease, but brain damage in patients with the disease occurs in several cortical and subcortical sites, making it difficult to determine the critical locus responsible for their cognitive deficits. The pathological changes in Alzheimer's disease are relatively selective, in that the ventromedial regions of the temporal and prefrontal lobes are particularly badly damaged (Pearson et al., 1985). Given that the effects of damage to these regions produce selective changes in cognitive function, notably impairments of memory, the damage to ventromedial cortical regions in Alzheimer's disease may also be responsible for the memory deficits typical of the condition. Furthermore, it has been suggested that the pathogen in Alzheimer's disease may extend along nerve fibers (Pearson et al., 1985; Saper et al., 1988), so that basal forebrain neurons projecting to or receiving afferents from the diseased cortical areas may be secondarily affected. In the normal brain, the efferent connections from the ventromedial limbic cortices to the basal forebrain may provide the substrate for the mnemonic information reflected in the responses of basal forebrain neurons.

Patients with Alzheimer's disease are not clearly deficient in their ability to regulate feeding and drinking, but are impaired in a range of cognitive functions. The responses of basal forebrain neurons reflect a coupling of motivational objectives and cognitive mechanisms underlying recognition of the identity and functional value of sensory stimuli, as opposed to a strictly motivational mechanism for the initiation and termination of feeding and drinking. This reinforcement-related neuronal ac- tivity presumably optimizes the operation of diverse cortical functions, including the limbic cortical regions with which it has bidirectional connections and which are important for memory function. Thus, damage to the basal forebrain might be expected to contribute to the deficits in memory tasks and the reduction in speed of information processing observed in $\mathrm{Alz}$ heimer's disease (Flicker et al., 1985) due to the loss of this optimizing mechanism. We suggest that the responses of basal forebrain neurons reflect a facilitation of sensory, motor, and mnemonic cortical functions and that the basal forebrain participates in many different types of learned and motivated behavior, including, but not restricted to, the cognitive control of feeding and drinking.

\section{References}

Aggleton, J. P., and M. Mishkin (1984) Projections of the amygdala to the thalamus in the cynomolgus monkey. J. Comp. Neurol. 222. 56-68.

Aigner, T., S. Mitchell, J. P. Aggleton, M. DeLong, R. Strubble, G. Wenk, D. Price, and M. Mishkin (1984) Recognition deficit in monkeys following neurotoxic lesions of the basal forebrain. Soc. Neurosci. Abstr. 10: 116.11.

Aigner, T. G., S. J. Mitchell, J. P. Aggleton, M. R. DeLong, R. G. Strubble, G. L. Wenk, D. L. Price, and M. Mishkin (1987) Effects of scopolamine and physostigmine on recognition memory in monkeys with ibotenic-acid lesions of the nucleus basalis of Meynert. Psychopharmacology 92: 292-300.

Albert, M., and M. Moss (1984) The assessment of memory disorders in patients with Alzheimer's disease. In Neuropsychology of Memory, L. R. Squire and N. Butters, eds., pp. 236-246, Guilford, New York.

Bachevalier, J., and M. Mishkin (1986) Visual recognition impairment follows ventromedial but not dorsolateral prefrontal lesions in monkeys. Behav. Brain Res. 20: 249-261.

Bondareff, W., C. Q. Mountjoy, and M. Roth (1982) Selective loss of neurones of origin of adrenergic projection to cerebral cortex (nucleus locus coeruleus) in senile dementia. Lancet 1: 783-784.

Bowen, D. M., C. B. Smith, P. White, and A. M. Davison (1976) Neurotransmitter-related enzymes and indices of hypoxia in senile dementia and other abiotrophies. Brain 99: 459-496.

Brown, M. W., F. A. W. Wilson, and I. P. Riches (1987) Neuronal evidence that inferomedial temporal cortex is more important than hippocampus in certain processes underlying recognition memory. Brain Res. 409: 158-162.

Brun, A., and E. Englund (1981) Regional pattern of degeneration in Alzheimer's disease: Neuronal loss and histopathological grading. Histopathology 5: 549-564.

Bruning, J. L., and B. L. Kintz (1977) Computational Handbook of Statistics, 2nd ed., Scott/Foresman, Glenview, IL.

Damasio, A. R., P. J. Eslinger, H. Damasio, G. W. Van Hoesen, and S. Cornell (1985a) Multimodal amnesic syndrome following bilateral temporal and basal forebrain damage. Arch. Neurol. 42: 252259.

Damasio, A. R., N. R. Graff-Radford, P. J. Eslinger, H. Damasio, and N. Kassal (1985b) Amnesia following basal forebrain lesions. Arch. Neurol. 42: 263-271.

Davies, P., and A. J. Maloney (1976) Selective loss of central cholinergic neurons in Alzheimer's disease. Lancet 11: 1430.

Dean, R. L., and R. T. Bartus (1985) Animal models of geriatric cognitive dysfunction: Evidence for an important cholinergic involvement. In Senile Dementia of the Alzheimer Type. J. Traber and W. H. Gispen, eds., pp. 269-282, Springer-Verlag, Berlin.

Drachman, D.A. (1977) Memory and cognitive function in man: Does the cholinergic system have a specific role? Neurology 27: 783-790.

Flicker, C., S. H. Ferris, T. Crook, R. T. Bartus, and B. Reisberg (1985) Cognitive function in normal aging and early dementia. In Senile Dementia of the Alzheimer Type, J. Traber and W. H. Gispen, eds., pp. 12-17, Springer-Verlag, Berlin.

Friedman, H. M., and N. Allen (1969) Chronic effects of complete limbic lobe destruction in man. Neurology 19: 679-689.

Gascon, G. G., and F. Gilles (1973) Limbic dementia. J. Neurol. Neurosurg. Psychiatry 36: 421-430.

Hedreen, J. C., R. G. Struble, P. J. Whitehouse, and D. L. Price (1984) 
Topography of the magnocellular basal forebrain system in human brain. J. Neuropathol. Exp. Neurol. 43: 1-21.

Horel, J. A., D. E. Pytko-Joiner, M. Voytko, and K. Salsbury (1987) The performance of visual tasks while segments of the inferotemporal cortex are suppressed by cold. Behav. Brain Res. 23: 29-42.

Humphrey, N. K. (1972) 'Interest' and 'Pleasure': Two determinants of a monkey's visual preferences. Perception 1: 395-416.

Jones, E. G., and T. P. S. Powell (1970) An anatomical study of converging sensory pathways within the cerebral cortex of the monkey. Brain 93: 793-820.

Jones, E. G., H. Burton, C. B. Saper, and L. W. Swanson (1976) Midbrain, diencephalic and cortical relationships of the basal nucleus of Meynert and associated structures in primates. J. Comp. Neurol. 167: 385-420.

Jurgens, U., and P. Muller-Preuss (1977) Convergent projections of different limbic vocalisation areas in the squirrel monkey. Exp. Brain Res. 29: 75-83.

Leichnetz, G. R., and J. Astruc (1977) The course of some prefrontal corticofugals to the pallidum, substantia innominata and amygdaloid complex in monkeys. Exp. Neurol. 54: 104-109.

Luria, A. R., Homskaya, E. D., Blinkov, S. M., and McD. Critchley (1967) Impaired selectivity of mental processes in association with a lesion of the frontal lobe. Neuropsychologia 5: 105-117.

Mair, W. G. P., E. K. Warrington, and L. Weiskrantz (1979) Memory disorder in Korsakoff's psychosis. Brain 192: 749-783.

Mesulam, M.-M., E. J. Mufson, A. I. Levey, and B. H. Wainer (1983) Cholinergic innervation of cortex by the basal forebrain: Cytochemistry and cortical connections of the septal area, diagonal band nuclei, nucleus basalis (substantia innominata) and hypothalamus in the rhesus monkey. J. Comp. Neurol. 214: 170-197.

Mesulam, M.-M., and E. J. Mufson (1984) Neural inputs into the nucleus basalis of the substantia innominata $(\mathrm{CH} 4)$ in the rhesus monkey. Brain 107: 257-274.

Mesulam, M.-M., A. D. Rosen, and E. J. Mufson (1984) Regional variations in cortical cholinergic innervation: Chemoarchitectonics of acetylcholinesterase-containing fibers in the Macaque brain. Brain 311: 245-258.

Mesulam, M.-M., L. Volicer, J. K. Marquis, E. J. Mufson, and R. C. Green (1986) Systematic regional differences in the cholinergic innervation of the primate cerebral cortex: Distribution of enzyme activities and some behavioral implications. Ann. Neurol. 19: 144-151.

Mishkin, M. (1978) Memory in monkeys severely impaired by combined but not separate removal of amygdala and hippocampus. Nature 273: 297-299.

Mishkin, M. (1982) A memory system in the monkey. Phil. Trans. R. Soc. London [Biol.] 298: 89-95.

Mishkin, M., and J. Delacour (1975) An analysis of short-tcrm visual memory in the monkey. J. Exp. Psychol.: Anim. Behav. Processes 1: 326-334.

Mora, F., E. T. Rolls, and M. J. Burton (1976) Modulation during learning of the responses of neurones in the lateral hypothalamus to the sight of food. Exp. Neurol. 53: 508-519.

Murray, E. A., and M. Mishkin (1986) Visual recognition in monkeys following rhinal cortical ablations combined with either amygdalectomy or hippocampectomy. J. Neurosci. 6: 1991-2003.

Nichelli, P., A. Baraldi, and G. Cappelli (1982) Hypernaetremic thirst deficiency and memory disorders following hypothalamic lesions. Arch. Psychiatr. Nervenkr. 231: 459-470.

Parent, A., and L. DeBellefeuille (1982) Organisation of efferent projections from the internal segment of the globus pallidus in the primate rcvealed by the fluorescence retrograde labeling method. Brain Res. 245: 201-213.

Pearson, R. C. A., M. M. Esiri, R. W. Hiorns, G. K. Wilcock, and T. P. S. Powell (1985) Anatomical correlates of the distribution of the pathological changes in the neocortex in Alzheimer's disease. Proc. Natl. Acad. Sci. USA 82: 4531-4534.

Perry, E. K., R. H. Perry, G. Blessed, and B. E. Tomlinson (1977) Necropsy evidence of central cholinergic deficits in senile dementia. Lancet 1: 189.

Ridley, R. M., H. F. Baker, B. Drewett, and J. A. Johnson (1985) Effects of ibotenic acid lesions of the basal forebrain on serial reversal learning in marmosets. Psychopharmacology 86: 438-443.

Ridley, R. M., T. K. Murray, J. A. Johnson, and H. F. Baker (1986) Learning impairment following lesion of the basal nucleus of Meynert in the marmoset: Modification by cholinergic drugs. Brain Res. 376 : $108-116$.

Rigdon, G. C., and J. H. Pirch (1986) Nucleus basalis involvement in conditioned neuronal responses in the rat frontal cortex. J. Neurosci. 6: $2535-2542$.

Rolls, E. T. (1986) Neuronal activity related to the control of feeding. In Feeding Behaviour: Neural and Humoral Controls, S. Ritter and C. D. Barnes, eds., pp. 163-190, Academic, New York.

Rolls, E. T. (1987) Information representation, processing and storage in the brain: Analysis at the single neuron level. In The Neural and Molecular Bases of Learning, J. P. Changeux and M. Konishi, eds., pp. 503-540, Wiley, Chichester.

Rolls, E. T., M. J. Burton, and F. Mora (1976) Hypothalamic neuronal responses associated with the sight of food. Brain Res. 111: 53-66.

Rolls, E. T., M. K. Sanghera, and A. Roper-Hall (1979) The latency of activation of neurones in the lateral hypothalamus and substantia innominata during feeding in the monkey. Brain Res. 164: 121-135.

Rolls, E. T., D. I. Perrett, A. W. Caan, and F. A. W. Wilson (1982) Neuronal responses related to visual recognition. Brain 105: 611646.

Room, P., F. T. Russchen, H. J. Groenwegan, and A. H . M. Lohman (1985) Efferent connections of the prelimbic (area 32) and the infralimbic (area 25) cortices: An anterograde tracing study in the cat. J. Comp. Neurol. 242: 40-55.

Russchen, F. T., D. G. Amaral, and J. L. Price (1985) The afferent connections of the substantia innominata in the monkey, macaca fascicularis. J. Comp. Neurol. 242: 1-27.

Saper, C. B., B. H. Wainer, and D. C. German (1988) Axonal and transneuronal transport in the transmission of neurological disease: Potential role in system degenerations, including Alzheimer's disease. Neuroscience 23: 389-398.

Scoville, W. B., and B. Milner (1957) Loss of recent memory after bilateral hippocampal lesions. J. Neurol. Neurosurg. Psychiatry 20: 11-22.

Squire, L. R., and R. Y. Moore (1979) Dorsal thalamic lesion in a noted case of chronic memory dysfunction. Ann. Neurol. 6: 503-506.

Talland, G. A., W. H. Sweet, and T. H. Ballantine, Jr. (1967) Amnesic syndrome with anterior communication artery aneurysm. J. Nerv. Ment. Dis. 145: 179-192.

Thorpe, S. J., E. T. Rolls, and S. Maddison (1983) The orbitofrontal cortex: Neuronal activity in the behaving monkey. Exp. Brain Res. 49: 93-115.

Travis, R. P., and D. L. Sparks (1969) Unitary responses and discrimination learning in the squirrel monkey: The globus pallidus. Physiol. Behav. 3: 187-196.

Voytko, M. L. (1985) Cooling orbitofrontal cortex disrupts matchingto-sample and visual discrimination learning in monkcys. Physiol Psychol. 13: 219-229.

Wallesch, C. W., H. H. Kornhuber, C. Kollner, H. C. Haas, and J. M. Hufnagl (1983) Language and cognitive deficits from medial and dorsolateral frontal lobe lesions. Arch. Psychiatr. Nervenkr. 233: 279296.

Whitehouse, P. J., A. W. Price, R. G. Struble, A. W. Clark, J. T. Coyle, and M. R. DeLong (1982) Alzheimer's disease and senile dementia: Loss of neurons in the basal forebrain. Science 215: 1237-1239.

Whitlock, D. G., and W. J. H. Nauta (1956) Subcortical projections from the temporal neocortex in Macaca mulatta. J. Comp. Neurol. 106: 184-207.

Wilson, F. A. W. (1989) Cortical and subcortical structures involved in recognition memory: Neurophysiological and anatomical studies. Int. J. Neurol. 21-22 (in press).

Wilson, F. A. W., and E. T. Rolls (1985) Reinforcement-related neuronal activity in the basal forebrain and amygdala. Soc. Neurosci Abstr. 15: 160.1

Wilson, F. A. W., and E. T. Rolls (1987) Habituation and stimulusspecific neuronal responses recorded from the amygdala of the monkey. Soc. Neurosci. Abstr. 17: 305.6.

Wilson, F. A. W., and E. T. Rolls (1989a) Neuronal responses related to reinforcement in the primate basal forebrain. Brain Res. (in press).

Wilson, F. A. W., and E. T. Rolls (1989b) Neuronal responses related to the novelty and familiarity of visual stimuli in the substantia innominata, diagonal band of Broca, and periventricular region of the primate basal forebrain. Exp. Brain Res. (in press).

Wilson, F. A. W., E. T. Rolls, S. Yaxley, S. J. Thorpe, G. V. Williams, 
and S. J. Simpson (1984) Responses of neurons in the basal forebrain of the behaving monkey. Soc. Neurosci. Abstr. 10:37.8.

Wilson, F. A. W., I. P. Riches, and M. W. Brown (1988) Neuronal activity in the inferomedial temporal cortex compared with that in the hippocampal formation: Implications for amnesia of medial temporal lobe origin. In Cellular Mechanisms of Conditioning and Behavioral Plasticity, C. D. Woody, D. L. Alkon, and J. L. McGaugh, eds., pp. 313-328, Plenum, New York.
Woodward, R. R., and P. L. Goldsmith (1964) Cumulative sum techniques. Mathematical and statistical techniques for industry. ICI monographs, No. 3. Oliver and Boyd, Edinburgh.

Zola-Morgan, S., and L. R. Squire (1985) Medial temporal lesions in monkeys impair memory on a variety of tasks sensitive to human amnesia. Behav. Neurosci. 99: 22-34. 